\title{
Leucine and mTORc1 act independently to regulate 2-deoxyglucose uptake in L6 myotubes
}

\author{
Qiong Yin ${ }^{1} \cdot$ John M. Brameld ${ }^{1}$ [D $\cdot$ Tim Parr $^{1} \cdot$ Andrew J. Murton ${ }^{1,2}$
}

Received: 5 September 2019 / Accepted: 8 February 2020 / Published online: 27 February 2020

(c) The Author(s) 2020

\begin{abstract}
Chronic mTORc1 hyperactivation via obesity-induced hyperleucinaemia has been implicated in the development of insulin resistance, yet the direct impact of leucine on insulin-stimulated glucose uptake in muscle cells remains unclear. To address this, differentiated L6 myotubes were subjected to various compounds designed to either inhibit mTORc1 activity (rapamycin), blunt leucine intracellular import (BCH), or activate mTORc1 signalling (3BDO), prior to the determination of the uptake of the glucose analogue, 2-deoxyglucose (2-DG), in response to $1 \mathrm{mM}$ insulin. In separate experiments, L6 myotubes were subject to various media concentrations of leucine $(0-0.8 \mathrm{mM})$ for $24 \mathrm{~h}$ before 2-DG uptake in response to insulin was assessed. Both rapamycin and BCH blunted 2-DG uptake, irrespective of insulin administration, and this occurred in parallel with a decline in mTOR, 4E-BP1, and p70S6K phosphorylation status, but little effect on AKT phosphorylation. In contrast, reducing leucine media concentrations suppressed 2-DG uptake, both under insulin- and non-insulin-stimulated conditions, but did not alter the phosphorylation state of AKT-mTORc1 components examined. Unexpectedly, 3BDO failed to stimulate mTORc1 signalling, but, nonetheless, caused a significant increase in 2-DG uptake under non-insulin-stimulated conditions. Both leucine and mTORc1 influence glucose uptake in muscle cells independent of insulin administration, and this likely occurs via distinct but overlapping mechanisms.
\end{abstract}

Keywords Insulin resistance $\cdot$ Obesity $\cdot$ Muscle $\cdot$ Glucose uptake $\cdot$ Leucine $\cdot$ Branched chain amino acids

\section{Introduction}

The global incidence of diabetes continues to increase unabated, with 451 million adults in 2017 estimated to have the condition, a number that is expected to increase to 693 million by 2045 (Cho et al. 2018). In attempting to delineate the aetiology of type- 2 diabetes mellitus (T2DM) which accounts for the majority of cases, it has been proposed that chronic increases in circulating concentrations of branched chain amino acids (BCAA) could contribute to the impaired ability of insulin to stimulate peripheral glucose disposal

Handling Editor: E. Closs.

John M. Brameld

john.brameld@nottingham.ac.uk

1 School of Biosciences, The University of Nottingham, Sutton Bonington campus, Loughborough, Leicestershire LE12 5RD, UK

2 Department of Surgery, University of Texas Medical Branch, Galveston, TX, USA in these patients (Newgard et al. 2009). Notably, increased blood concentrations of BCAA have been observed in both insulin resistant and obese individuals (Caballero et al. 1988; Felig et al. 1969), with the concentrations of various BCAArelated metabolites showing a significant linear relationship with measures of insulin resistance in humans (Newgard et al. 2009). Moreover, in animal models of obesity, the genetic or pharmacological manipulation of the branched chain ketoacid dehydrogenase complex so as to aid clearance of circulating BCAAs via increased hepatic uptake causes improvements in both insulin sensitivity and glucose tolerance (White et al. 2018).

BCAA, in particular the amino acid leucine, are widely known to induce profound metabolic effects on skeletal muscle, a major site of glucose disposal in the body. Leucine is a potent stimulator of protein synthesis within muscle cells, a process achieved through activation of the kinase mammalian target of rapamycin (mTORc1), which, in turn, drives translation initiation as a consequence of the phosphorylation of downstream targets 4E-BP1 and p70S6K (see Murton et al. 2008). In recent years, it has also been suggested 
that mTORc1 is able to phosphorylate upstream targets of the insulin signalling pathway in muscle, leading to inhibition of the pathway, and in turn impaired translocation of the GLUT4 glucose transporter to the plasma membrane (Yoneyama et al. 2018). Thus, it has been proposed that a potential mechanism by which obesity-induced increases in blood BCAA concentrations could lead to the development of insulin resistance is through the chronic activation of mTORc1 suppressing insulin signalling in skeletal muscle.

In contrast to this purported mechanism, we have found that muscle mTORc1 signalling and its response to exogenous amino acids is not impaired in obese men, despite clear evidence for the presence of insulin resistance (Murton et al. 2015). Likewise, it has been reported that both acute and chronic systemic inhibition of mTORc1 function induces insulin resistance in rodents (Deblon et al. 2012; Kleinert et al. 2014), and the administration of leucine or mixed amino acids can increase insulin-stimulated glucose uptake (Kleinert et al. 2011; Li et al. 2013). In light of these findings, debate remains surrounding the contribution of BCAA-stimulated mTORc1 hyperactivation in the pathophysiology of peripheral insulin resistance.

A potential explanation for the discord between findings to date is the challenge associated with studying the impact of amino acids on tissue metabolism in vivo. As noted by others (White and Newgard 2019), supplementing with individual amino acids or manipulating systemic mTORc1 function also impacts metabolism in distal tissues, potentially underpinning the inconsistent observations seen between studies. Thus, to circumvent these issues, the aim of the current study was to delineate the role of leucine on glucose uptake in cultured muscle cells, utilizing multiple strategies to manipulate leucine availability and mTORc 1 function so as to ascertain their contribution to insulin-stimulated glucose uptake.

\section{Materials and methods}

\section{Materials}

Culture media (high-glucose Dulbecco's Modified Eagle's Media, DMEM), 3BDO, and BCH were obtained from Sigma-Aldrich (Poole, UK.) DMEM without glucose and L-leucine was ordered from Cell Culture Technologies (Ticino, Switzerland). L-leucine free medium (DMEMLM, 30,030) and horse serum were ordered from Thermo Fisher (Loughborough, UK). Heat inactivated Fetal bovine serum (FBS) and Penicillin/Streptomycin were obtained from Gibco by Life technologies (Loughborough, UK). Rapamycin was purchased from Cell Signalling Technologies (London, UK). Antibodies: rabbit anti mTOR antibody (\#2972), rabbit anti-phospho-mTOR (Ser2448; \#2971), rabbit anti-phospho-4E-BP1 (Thr37/46; \#9459), rabbit anti-4E-BP1 (\#9452), rabbit anti-phospho-p70 S6 Kinase (Thr389; \#9205), rabbit anti-p70 S6 Kinase (\#9202), rabbit anti-phospho-Akt (Ser473,\#9271), rabbit anti-Akt (\#9272), rabbit anti- $\beta$-Actin Antibody (\#4967), anti-rabbit IgG, and HRP-linked Antibody (\#7074) were all purchased from Cell Signalling Technologies (London, UK). Glucose Uptake$\mathrm{Glo}^{\mathrm{TM}}$ Assay kit was purchased from Promega (Southampton, UK).

\section{Cell culture}

L6 myoblasts were cultured in growth medium consisting of high-glucose Dulbecco's Modified Eagle Media (DMEM), supplemented with $10 \%(\mathrm{v} / \mathrm{v})$ fetal bovine serum, and $1 \%$ $(\mathrm{v} / \mathrm{v})$ penicillin and streptomycin, until approximately $70-80 \%$ confluent. High-glucose DMEM was utilized in response to our observations that L6 cells cultured under low-glucose DMEM (5.6 mM) failed to demonstrate robust uptake of the glucose analogue 2-deoxyglucose, in response to $100 \mathrm{mM}$ insulin administration (data not shown). After the L6 myoblasts had reached the required level of confluence, they were switched to differentiation medium consisting of DMEM supplemented with 2\% (v/v) horse serum and $1 \%(\mathrm{v} / \mathrm{v})$ penicillin and streptomycin, causing the formation of myotubes. The day that L6 myoblasts were switched to differentiation media was labelled as 'day 0 ', and differentiation media were replaced every 2 days.

At days 5 or 6 , a time period which we have shown sufficient to induce myotube formation (Hurley et al. 2006), the differentiation media were removed and replaced with fresh DMEM containing 1\% (v/v) penicillin and streptomycin but devoid of serum. Cells were maintained under the serum starvation protocol for $24 \mathrm{~h}$ before the impact of various investigatory drugs studied. To investigate the impact of mTORc1 function on glucose uptake, rapamycin (100 nM), or 3-Benzyl-5-((2-nitrophenoxy)methyl)-dihydrofuran-2(3H)-one (3BDO; $60 \mu \mathrm{M})$, both initially dissolved in dimethylsulfoxide (DMSO), were administered to myotubes for a period of 1 (rapamycin only) or $4 \mathrm{~h}$ (both) to either inhibit or stimulate mTORc1 activity, respectively, and compared to controls also containing 1.2\% DMSO. In separate experiments, to establish the direct impact of leucine on insulin-stimulated glucose uptake, myotubes were treated following $24 \mathrm{~h}$ serum starvation with either the nonmetabolizable leucine analogue, b(-)2-amino-bicyclo[2,2,1] heptane-2-carbocyclic acid $(\mathrm{BCH} ; 25 \mathrm{mM})$ or leucine (0-0.8 mM, where $0.8 \mathrm{mM}$ represents the leucine concentration of standard DMEM), with both dissolved in the media and administered for 4 or $24 \mathrm{~h}$, respectively.

After their respective treatments, the subsequent impact of the various compounds and leucine concentrations on 
insulin-stimulated glucose uptake and mTORc1 signalling were assessed as described below.

\section{Glucose uptake assay}

Glucose uptake in L6 myotubes was assessed using a commercial kit that involves the determination of 2-deoxyglucose-6-phosphate (2DG6P) concentrations in cells following 2-deoxyglucose administration. In short, following treatment with the test compound, half of the treated wells were incubated at $37^{\circ} \mathrm{C}$ for $1 \mathrm{~h}$ in the presence of $1 \mathrm{mM}$ insulin. After the allotted time, the media were removed and the cells were washed in PBS, followed by the addition of $0.1 \mathrm{mM}$ 2-deoxyglucose in PBS to all wells. Cells were incubated for 30 min at $25{ }^{\circ} \mathrm{C}$, after which $2 \mathrm{DG} 6 \mathrm{P}$ concentrations were measured according to the manufacturer's instructions using a luminescence plate reader (Fluostar, Omega).

\section{Intracellular free branched chain amino acid (BCAA) concentrations}

A commercial colorimetric assay kit for the determination of BCAA concentrations was utilized to assess free intracellular BCAA concentrations (BioVision). Following treatment with the test compound, cells were collected in $50 \mu \mathrm{l}$ of assay buffer and subsequently lysed by sonication for 10-15 s. Afterwards, samples were centrifuged at $14,000 \mathrm{rpm}$ for $10 \mathrm{~min}$ at $4{ }^{\circ} \mathrm{C}$ to remove cellular debris and other insoluble material. $20 \mu \mathrm{l}$ of the resultant supernatant which contained the intracellular free amino acids was diluted to $50 \mu \mathrm{l}$ with assay buffer and analysed according to the manufacturer's instructions, with the resultant colour product detected using a microplate reader set at a wavelength of $450 \mathrm{~nm}$ (Fluostar, Omega). The concentration obtained through use of this approach equated to the sum of the concentration of the three individual BCAA: leucine, isoleucine, and valine.

\section{Western blot analysis}

To establish the impact of the various treatments on mTORc1 signalling, L6 myotubes grown in 6-well plates were lysed at the end of the treatment with the test compound using ice-cold lysis buffer (20\% v/v glycerol, $12.5 \%$ v/v 1 M Tris-HCL (pH 6.8), 4\% w/v SDS, $10 \% 1$ M DTT, and $0.004 \% \mathrm{w} / \mathrm{v}$ bromophenol blue), containing both phosphatase and protease inhibitors (Roche). Protein concentrations were determined using the commercial 2D Quant kit (GE Healthcare Life Sciences, Buckinghamshire, UK) and samples adjusted to a constant final concentration of 1 or $2 \mu \mathrm{g} / \mu \mathrm{l}$ (dependent upon experiment) using lysis buffer. Afterwards, $15 \mu \mathrm{l}$ of each sample was separated by electrophoresis on a 4-15\% SDS-PAGE gel and transferred to PVDF membranes (GE Healthcare) using the established techniques (Murton et al. 2009). Afterwards, membranes were blocked by incubating in 5\% (w/v) bovine serum albumin (BSA). The abundance and phosphorylation state of individual proteins were assessed using commercial primary antibodies diluted 1:1000 in 5\% w/v BSA, HRP-linked secondary antibody diluted 1:5000 with 5\% w/v BSA, and visualized by enhanced chemiluminescence (ECL; GE Healthcare, Buckinghamshire, UK). When determining both the degree of phosphorylation and total protein abundance of the same target protein, the membrane was first probed by use of the phosphorylation-specific antibody, before being stripped with a commercial stripping buffer (Thermo Fisher), and re-probed with the non-phosphorylation specific version of the antibody. Blots were analysed using the ChemiDoc MP Imaging System (Bio-Rad, CA, USA) and associated Image Lab 6.0.1 Software (Bio-Rad, CA, USA).

\section{Statistics}

Except where stated, when considering the effect of insulin and rapamycin on 2-deoxyglucose uptake and mTORc1 signalling (Fig. 1), a two-way ANOVA was performed to test for statistical significance. In all other cases, insulin- and non-insulin-stimulated experiments were performed in separate batches, and as such data were analysed by use of a oneway ANOVA statistical test. All statistical tests were performed using the software, Prism (v 8.2.0, GraphPad). When a significant main effect was observed (one-way ANOVA) or interaction (two-way ANOVA), a post hoc test (Tukey) was performed to identify statistically significant differences between groups. A value of $p<0.05$ was considered statistically significant. Data are presented as means \pm standard error of the mean (SEM).

\section{Results}

\section{Rapamycin-induced inhibition of mTORc1 signalling blun}

\section{ts glucose uptake in L6 myotubes, irrespective of insulin administration}

It has previously been shown that a discordance exists in the ability of insulin to stimulate glucose uptake in muscle cell lines of murine and rodent origin (Sarabia et al. 1990). Thus, to confirm that the cultured L6 myotubes are sensitive to the actions of insulin and represent an appropriate model to investigate glucose uptake in muscle cells, L6 myotubes were administered $100 \mathrm{nM}$ insulin, a dose known to induce robust increases in glucose uptake (Yap et al. 2007). Treatment with insulin for $1 \mathrm{~h}$ resulted in a doubling in the uptake 

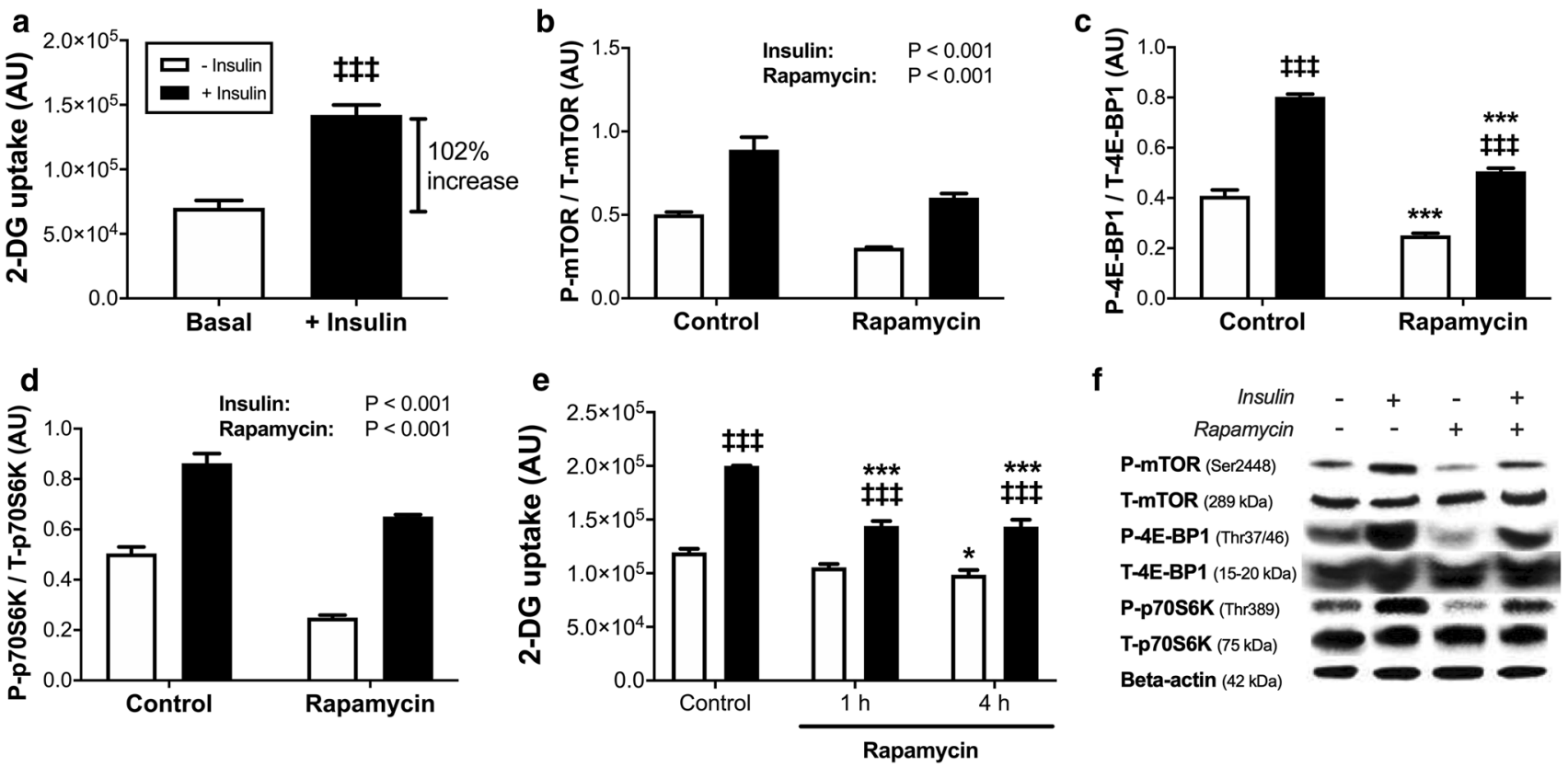

Fig. 1 Rapamycin inhibits mTORc1 signalling and 2-deoxyglucose (2-DG) uptake in L6 myotubes under both insulin- and non-insulinstimulated conditions. 2-DG uptake (a) in response to $1 \mathrm{mM}$ insulin, administered for $1 \mathrm{~h}$. Impact of $1 \mathrm{~h}$ pre-treatment with $100 \mathrm{nM}$ rapamycin on the phosphorylation state of mTOR (b), 4E-BP1 (c), and p70S6K (d) in the presence or absence of $1 \mathrm{mM}$ insulin $(n=3$ per group). e Impact of 1 and $4 \mathrm{~h}$ pre-treatment with $100 \mathrm{nM}$ rapamycin on 2-DG uptake in the presence or absence of $1 \mathrm{mM}$ insulin $(n=6$ per

of the glucose analogue compared to control, confirming the suitability of this model (Fig. 1a, $p<0.001$, unpaired $t$ test).

Next, to confirm the effect of insulin administration on mTORc1 signalling, and to ascertain whether mTORc1 activity in part contributes to insulin-dependent glucose uptake in muscle cells, mTORc1 activity was blunted using the established inhibitor, rapamycin, at a concentration known to inhibit mTORc1 signalling (Deblon et al. 2012). This was performed under both insulin and non-insulinstimulated conditions. As expected, insulin administration resulted in significant increases in the phosphorylation state of mTOR and its downstream targets 4E-BP1 and p70S6K (main effect of insulin: $p<0.001$; Fig. 1b-d and f), while treatment with $100 \mathrm{nM}$ rapamycin for $1 \mathrm{~h}$ induced decreased phosphorylation of mTOR and its downstream targets, irrespective of insulin treatment ( $p<0.05$; Fig. 1b-d). Likewise, rapamycin treatment blunted the ability of insulin to stimulate uptake of the glucose analogue by $52 \%$, which persisted even when the period of rapamycin treatment was extended by a further $3 \mathrm{~h}$ (Fig. 1e). Collectively, these findings suggest that the dose of rapamycin utilized in these experiments was effective at impairing insulin-stimulated mTORc1 signalling and resulted in a blunted ability of insulin to induce cellular glucose uptake. Somewhat unexpectedly, a similar, albeit group). f Representative western blot images. $* p<0.05 ; * * * p<0.001$ significantly different from respective control. $\$ \neq \neq p<0.001$ significantly different from non-insulin treated group. b-e Post hoc tests were performed only when a significant interaction between terms was observed $(p<0.05)$; otherwise, results for main effects are reported. Data are presented as means \pm SEM. Con control, Rap rapamycin

more modest ability of rapamycin to suppress mTORc1 signalling and 2-deoxyglucose uptake was also observed in the absence of insulin (Fig. 1b-e).

\section{BCH significantly impairs the ability of insulin to stimulate mTORc1 signalling and 2-deoxyglucose uptake in L6 myotubes, independent of insulin signalling}

Inhibiting the action of mTORc1, even transiently, is known to lead to an increase in intracellular amino acid concentrations due to decreased sequestering of amino acids in nascently synthesized proteins (Mubeen et al. 2018). Thus, it is permissible that the inhibitory effect of rapamycin on 2-deoxyglucose uptake is the result of increased intracellular leucine concentrations as opposed to impaired mTORc1 function per se. To discount this possibility, the non-metabolizable analogue of L-leucine, $\mathrm{BCH}$, was used to inhibit cellular leucine uptake via competing for the LAT1 transporter. Given leucine's key role in mediating increased mTORc1 activity in response to amino acid provision (Wolfson et al. 2016), acute $\mathrm{BCH}$ administration represents an alternative strategy to reduce mTORc1 function without increasing leucine concentrations. Notably, when comparing the effect of 
$4 \mathrm{~h}$ treatment of $100 \mathrm{nM}$ rapamycin with $25 \mathrm{mM} \mathrm{BCH}$, only the former affected intracellular-free BCAA concentrations, where a $78 \%$ increase was observed ( $p<0.001$; Fig. 2a).

To confirm that $\mathrm{BCH}$ does indeed inhibit mTORc1 activity, L6 myotubes were incubated for $4 \mathrm{~h}$ with either $100 \mathrm{nM}$ rapamycin or $25 \mathrm{mM} \mathrm{BCH}$, prior to $1 \mathrm{~h}$ treatment with or without insulin. Utilizing this approach, the phosphorylation state of mTOR, 4E-BP1, and p70S6K was suppressed with use of either inhibitor, both under insulin- and non-insulinstimulated conditions (Fig. 2b-d and g). However, with the exception of 4E-BP1, the suppressive effect of rapamycin on mTORc1 signalling was greater than that of $\mathrm{BCH}$. Despite this, $\mathrm{BCH}$ administration suppressed 2-deoxyglucose uptake to a greater degree than that of rapamycin, with $\mathrm{BCH}$ suppressing uptake of the glucose analogue below basal levels and fully preventing the stimulatory effects of insulin on 2-deoxyglucose uptake (Fig. 2e). Collectively, these findings suggest that the inhibitory effect of $\mathrm{BCH}$ on 2-deoxyglucose uptake occurs, at least in part, through a mechanism independent of mTORc1. To ascertain whether this could be due to inhibition of AKT phosphorylation, a key mediator of insulin-mediated GLUT4 translocation, the phosphorylation state of AKT Ser473 was examined. Neither rapamycin nor $\mathrm{BCH}$ administration affected the stimulatory effect of insulin on AKT phosphorylation (Fig. 2f), suggesting that the detrimental impact of $\mathrm{BCH}$ on glucose uptake was not mediated by a change in AKT activity.

\section{Leucine availability modulates glucose uptake in L6 myotubes, independent of mTORc1 and AKT signalling}

To determine whether leucine availability impacted on glucose uptake, L6 myotubes were subjected to different leucine concentrations $(0.0-0.8 \mathrm{mM})$ for a period of $24 \mathrm{~h}$, and the impact on mTORc1 signalling and 2-deoxyglucose uptake in the presence and absence of insulin assessed.

Under the conditions studied, modulating leucine availability had no impact on the phosphorylation state of various mTORc1 signalling proteins under either basal or insulin-stimulated conditions (Fig. 3a-c and f). Similarly, the phosphorylation state of AKT was unaffected by decreasing leucine media concentrations (Fig. 3e). While modulating media leucine concentrations had no measurable effect on the AKT-mTORc1 signalling axis, there was a significant impact on 2-deoxyglucose uptake (Fig. 3d). In the absence of insulin, a dose-dependent increase in glucose uptake with media leucine concentrations was observed. Moreover, the ability of insulin to stimulate 2-deoxyglucose uptake appeared impaired by leucine deprivation.
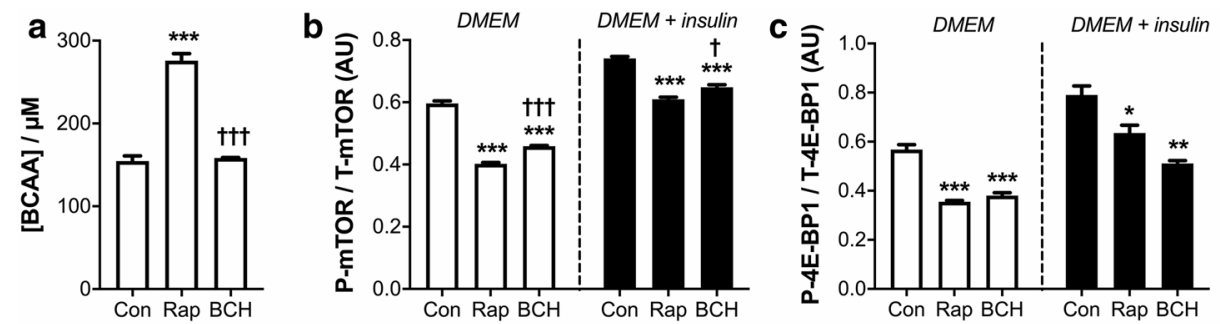

e

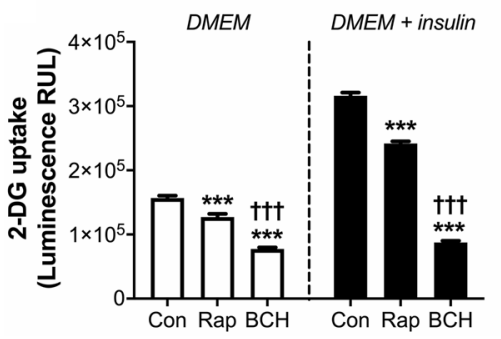

f

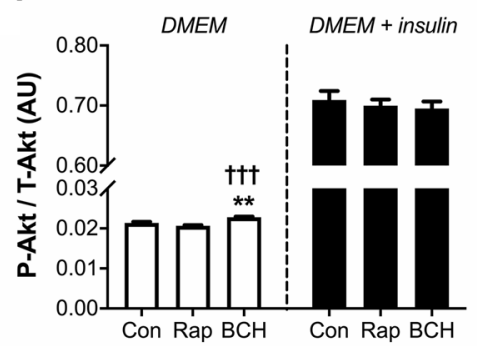

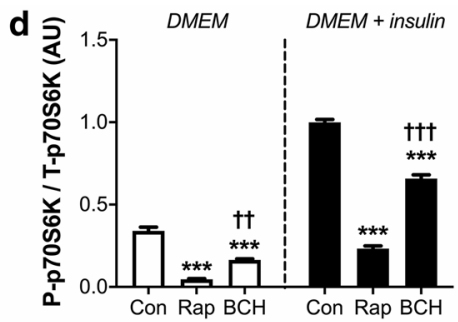

g

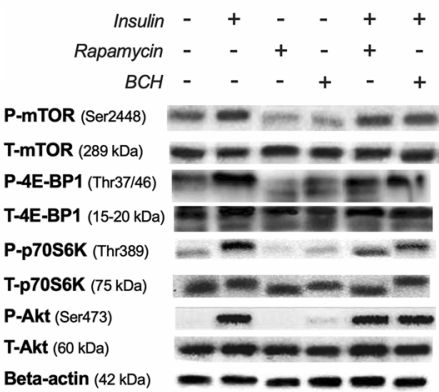

Fig. $2 \mathrm{BCH}$ decreases mTORc1 signalling and completely blunts the ability of insulin to stimulate 2-deoxyglucose (2-DG) uptake in L6 myoblasts, in an AKT-independent manner. Impact of $4 \mathrm{~h}$ pretreatment with $100 \mathrm{nM}$ rapamycin or $25 \mathrm{mM} \mathrm{BCH}$ on intracellular concentrations of free branched chain amino acids (BCAA; a). Effect of the same treatments on the phosphorylation state of mTORc1 signalling components (b-d) and AKT (f) in the presence or absence of $1 \mathrm{mM}$ insulin ( $n=3$ per group). e Impact of rapamycin or $\mathrm{BCH}$ pre-treatment on insulin- and non-insulin-stimulated 2-DG uptake ( $n=6$ per group). g Representative western blot images. ${ }^{*} p<0.05$; $* * p<0.01 ; * * * p<0.001$, significantly different from respective control. $\dagger p<0.05$; $\dagger \dagger p p<0.001$, significantly different from rapamycin. Data are presented as means \pm SEM. $B C H$ b(-)2-amino-bicyclo[2,2,1] heptane-2-carbocyclic acid, Con control, DMEM Dulbecco's modified eagle medium, Rap rapamycin 

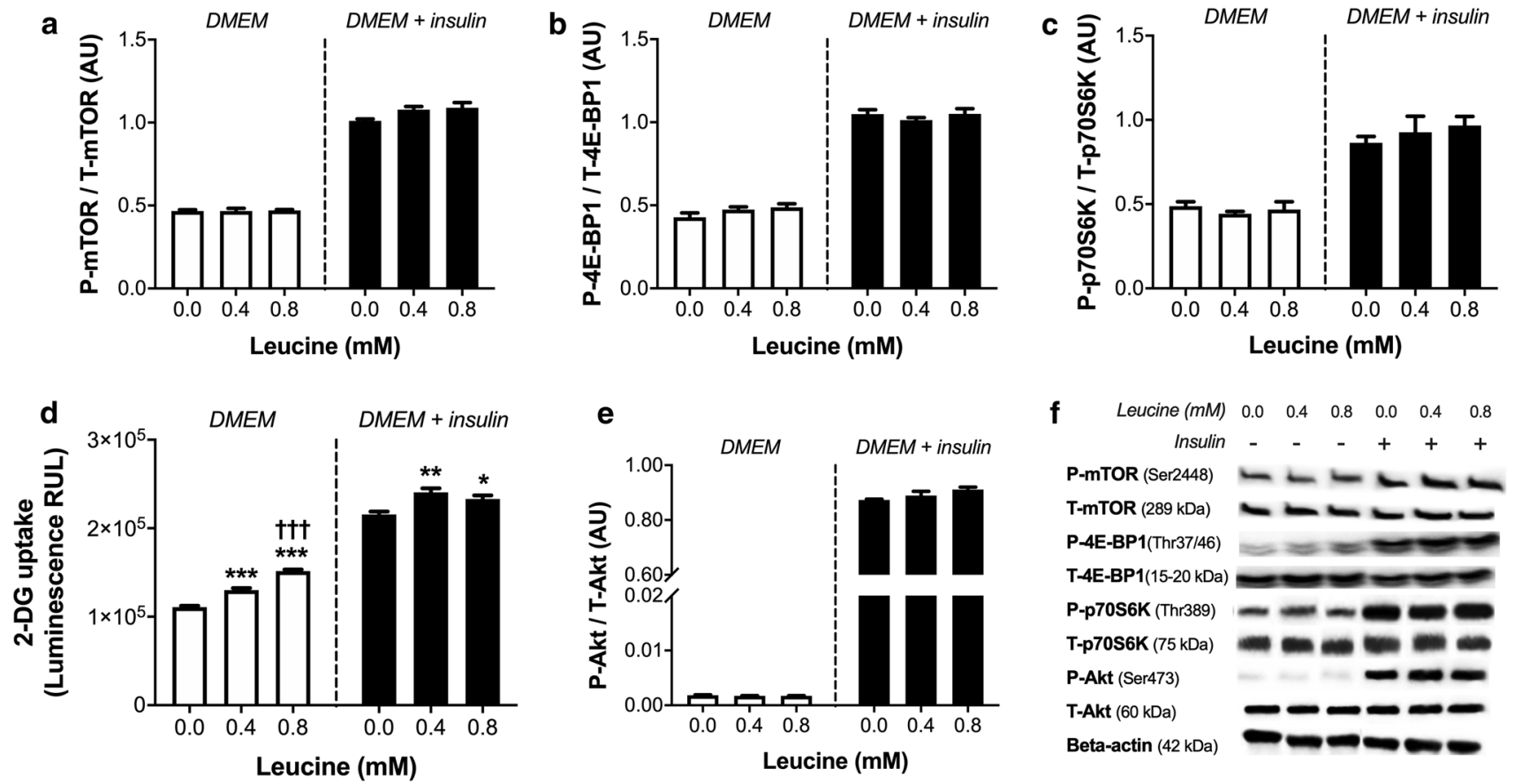

Fig. 3 Modest changes in leucine availability affect 2-deoxyglucose (2-DG) uptake in L6 myotubes under both insulin- and non-insulinstimulated conditions, and occurs in the absence of changes in AKTmTORc1 signalling. Impact of $24 \mathrm{~h}$ treatment with various concentrations of leucine on the phosphorylation state of mTORc1 signalling components (a-c) and AKT (e) in the presence or absence of $1 \mathrm{mM}$ insulin ( $n=3$ per group). d Impact of leucine media concentra-

\section{$3 B D O$ reduced $m T O R$ phosphorylation, leading to an enhancement of 2-deoxyglucose uptake in the absence of insulin}

Given that chronic mTORc1 activation has been proposed as a contributor to impaired muscle glucose uptake in insulinresistant individuals (Newgard et al. 2009), we wanted to investigate what impact mTORc1 hyperactivation had on the ability of insulin to stimulate 2-deoxyglucose uptake in myotubes. The compound 3BDO has been described as a potent mTORc1 activator, and its effectiveness confirmed in a number of cell types (Ge et al. 2014; Peng et al. 2014; Sheng et al. 2017; Wang et al. 2016). Thus, utilizing 3BDO represented a unique pharmacological approach to assess mTORc1 activity on glucose uptake in muscle cells.

L6 myotubes were exposed for $4 \mathrm{~h}$ to either $100 \mathrm{nM}$ rapamycin or $60 \mu \mathrm{M}$ of the mTORc1 activator to confirm the effect of mTORc1 hyperactivity on insulin-stimulated glucose uptake. Contrary to expectations, 3BDO decreased the phosphorylation of mTOR and p70S6K in the absence of insulin compared to control (Fig. 4a-c and f; $p<0.001$ ). Similarly, the phosphorylation state of 4E-BP1 and P70S6K in response to insulin was below that seen with control when treated with 3BDO. In spite of mTORc1 signalling being tions on insulin- and non-insulin-stimulated 2-DG uptake $(n=6$ per group). f Representative western blot images. $* p<0.05$; $* p<0.01$; $* * * p<0.001$, significantly different from $0 \mathrm{mM}$ leucine treatment. $\dagger+\dagger p<0.001$, significantly different from $0.4 \mathrm{mM}$ leucine treatment. Data are presented as means \pm SEM. DMEM Dulbecco's modified eagle medium

impaired by 3BDO treament, the compound significantly increased 2-deoxyglucose uptake in the absence of insulin $(p<0.001)$; albeit, this effect was reversed when examined under insulin-stimulated conditions (Fig. 4d). While a discord in the ability of 3BDO to stimulate 2-deoxyglucose under insulin- and non-insulin-stimulated conditions was readily apparent, the mTORc1 activator significantly increased the phosphorylation status of AKT compared to control, irrespective of insulin administration $(p<0.001$; Fig. 4e). Unexpectadly, a modest, albeit significant $(p<0.05)$ increase in AKT phoshorylation status was also observed with rapamycin treatment under insulin-stimulated conditions.

\section{Discussion}

A definitive role for obesity-induced hyperleucinaemia in the induction of type- 2 diabetes has remained controversial, due in part to inconsistent reports on the impact of leucine on insulin-stimulated glucose uptake in skeletal muscle. To provide much needed clarity on the role of leucine in regulating glucose uptake in muscle cells, we have examined the impact of modifying leucine availability and mTORc1 function on 
a

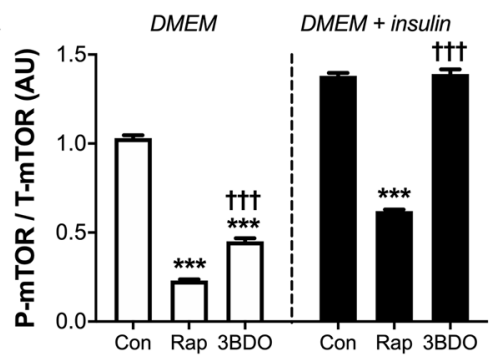

d

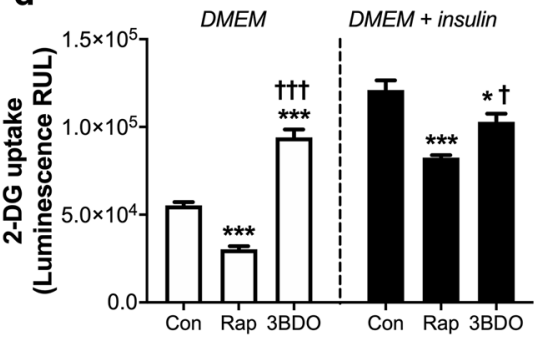

b

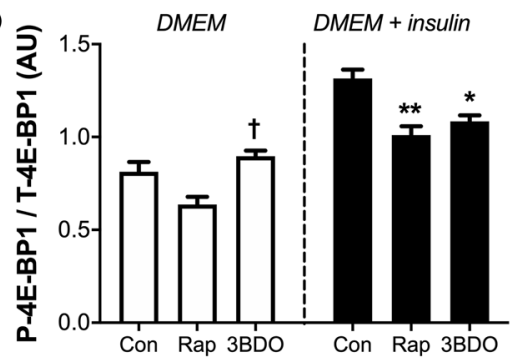

e

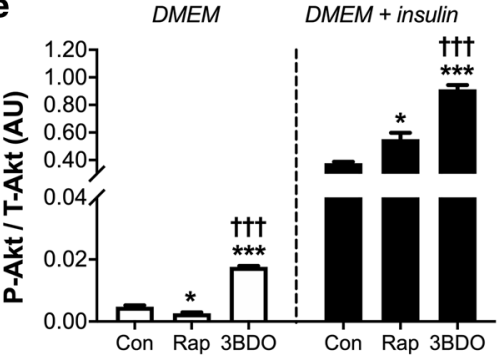

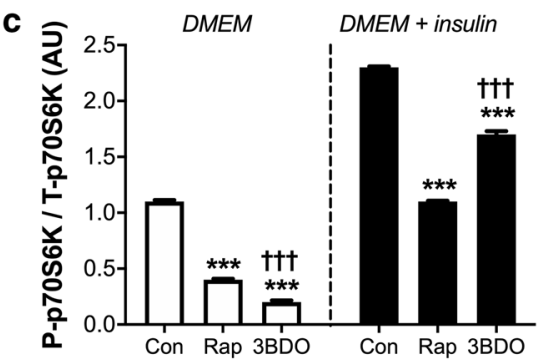

$\mathbf{f}$

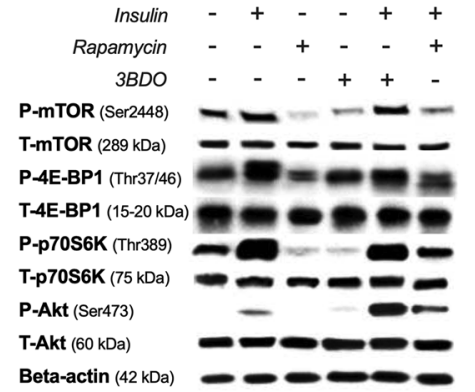

Fig. 4 Treatment with the mTORc1 activator 3BDO inhibits components of mTORc1 signalling, but has opposing effects on 2-deoxyglucose (2-DG) uptake dependent on the presence of insulin. Impact of $4 \mathrm{~h}$ pre-treatment with either $100 \mathrm{nM}$ rapamycin or $60 \mu \mathrm{M} 3 \mathrm{BDO}$ on the phosphorylation state of mTORc1 signalling components (a-c) and AKT (e) in the presence or absence of $1 \mathrm{mM}$ insulin ( $n=3$ per group). d Impact of rapamycin or 3BDO pre-treat- ment on insulin- and non-insulin-stimulated 2-DG uptake ( $n=6$ per group). f Representative western blot images. ${ }^{*} p<0.05$; $* * p<0.01$; $* * * p<0.001$, significantly different from respective control. $\dagger p<0.05 ; \dagger \dagger \dagger p<0.001$, significantly different from rapamycin. Data are presented as means \pm SEM. 3BDO 3-Benzyl-5-((2-nitrophenoxy) methyl)-dihydrofuran-2(3H)-one, Con control, DMEM Dulbecco's modified eagle medium, Rap rapamycin insulin-stimulated glucose uptake in myotubes. Utilizing this approach, we demonstrate that leucine availability appears to play an essential role in glucose uptake in L6 myotubes, irrespective of insulin provision. Moreover, we show that leucine's ability to modulate glucose uptake can occur independent of changes in mTORc1 signalling status. Finally, we demonstrate that the mTORc1 activator, 3BDO, can modulate muscle cell glucose uptake, potentially representing an interesting pharmacological strategy to enhance non-insulin dependent glucose uptake in insulin resistant states.

While the impact of exogenous leucine on muscle glucose uptake has been widely investigated, a consensus on its main effects has yet to be reached. Indeed, while numerous studies have demonstrated that leucine administration impairs the ability of insulin to stimulate glucose uptake and worsens glucose tolerance in animals (Balage et al. 2011; Deshmukh et al. 2009; Iwanaka et al. 2010; Moghei et al. 2016; Saha et al. 2010), this is far from universally observed. Rats administered a diet for 5 weeks containing $15 \%$ protein supplemented with $4.5 \%$ leucine have been shown to experience a significant increase in insulin-stimulated glucose transport in epitrochlearis muscle when assessed ex vivo (Balage et al. 2011). Notably, this is in spite of an overall fall in whole-body insulin sensitivity as determined by oral glucose tolerance test. Similarly, application of media containing high leucine concentrations $(5-10 \mathrm{mM})$ has been shown to result in significant increases in 2-deoxyglucose uptake in $\mathrm{C} 2 \mathrm{C} 12$ myotubes, even in the absence of insulin (Doi et al. 2003). In contrast, others have shown that the ability of leucine to facilitate glucose uptake in myotubes derived from rat primary skeletal muscle cells occurs only when co-administered with insulin (Liu et al. 2014). While our findings are unable to definitely address these contradictory reports, they provide further support for leucine playing an important role in glucose uptake in muscle cells, under both insulin- and non-insulin-stimulated conditions. Utilizing various approaches to either suppress leucine extracellular availability, intracellular import, or leucine-regulated signalling mechanisms, 2-deoxyglucose uptake was consistently blunted irrespective of insulin administration, underpinning its crucial and direct role in regulating muscle cell glucose metabolism. Moreover, by employing techniques that have allowed mTORc1 activity and leucine availability to be manipulated in isolation of each other, we demonstrate that leucine and mTORc1 act independently to regulate 2-deoxyglucose uptake in muscle cells.

The notion that leucine plays a permissive role in the uptake of glucose in muscle cells has important implications concerning the development of approaches to treat type-2 diabetes. Growing emphasis has been placed on the concept of manipulating branched chain amino acids in the diet to improve insulin sensitivity in at risk populations (Xiao et al. 
2011). While leucine deprivation has been found to improve muscle insulin signalling in rodents (Xiao et al. 2011), the ability of the approach to increase muscle insulin sensitivity remains uncertain. Utilizing a leucine analogue as a competitive inhibitor for leucine, thereby limiting cellular uptake of the amino acid via its transporter, we demonstrate that leucine import likely plays a crucial process in driving insulin-stimulated glucose uptake. While the exact mechanism by which this occurs is unclear, it is not without precedent. Muscle-specific knockout of the LAT1 transporter in mice has been shown to impair whole-body glucose uptake in response to a glucose challenge (Poncet et al. 2014). Moreover, the authors' demonstration of the importance of LAT1 in the activation of muscle mTORc1 signalling by exogenous leucine viewed in conjunction with our findings that rapamycin blunts glucose uptake in muscle cells, which highlights one possible mechanism by which dietary leucine opposes the development of insulin resistance in muscle.

While, in the current study, the inhibitory action of rapamycin on glucose uptake was consistently observed and occurred independent of insulin administration, others have argued that any impact of leucine on muscle glucose uptake occurs in a purely mTORc1 independent manner. In isolated rodent muscle, pre-treatment with rapamycin was unable to prevent the stimulatory effects of leucine on 2-deoxyglucose uptake, albeit the same study failed to demonstrate that mTORc1 was inhibited by rapamycin at the dose used, questioning the validity of their findings (Nishitani et al. 2002). In contrast, and in full agreement with the data which we present, others have shown that rapamycin blocks the beneficial effect of leucine on insulin-stimulated glucose uptake in rat primary skeletal muscle cells (Liu et al. 2014). Similarly, when alternative inhibition strategies have been investigated aimed at blocking the action of PI3K, a kinase upstream of mTORc1 in canonical pathways, insulin-stimulated glucose uptake in cultured muscle cells was impaired (Liu et al. 2014). Moreover, this effect was partially rescued by leucine administration, demonstrating that insulin and leucine's beneficial impact on glucose uptake occurs via different mechanisms. In support of this notion, whilst we observed that AKT phosphorylation was significantly increased in response to insulin administration, this was not blunted by declining leucine media concentrations or the administration of either rapamycin or $\mathrm{BCH}$.

Curiously, while leucine availability is widely acknowledged as a potent activator or mTORc1 signalling, at the concentrations studied in the current report $(0.0-0.8 \mathrm{mM})$, modulating leucine media concentrations failed to impact the examined components of mTORc1 signalling. We believe that there are two plausible explanations for this observation. First, in the previous studies designed to elicit increased mTORc1 signalling activity, higher media concentrations of leucine were typically utilized ( 2 to $5 \mathrm{mM})$. Thus, it is permissible that the maximum concentration used in the current study $(0.8 \mathrm{mM})$, which is equivalent to the concentration of leucine found in DMEM, was insufficient to stimulate mTORc1 activity. Conversely, the ability of leucine to stimulate mTORc1 activity has been shown to be transient, returning to basal levels despite leucine availability remaining elevated (Gran and Cameron-Smith 2011). Therefore, it is possible that at the timepoint examined ( $24 \mathrm{~h})$, any increase in mTORc1 signalling had been missed. Regardless of the underlying cause for the lack of observed effect of leucine on mTORc1 signalling, our data demonstrate the crucial role that leucine availability plays in cellular glucose uptake in muscle cells, under both insulin- and non-insulinstimulated conditions.

While our data robustly demonstrate that leucine mediates glucose uptake in skeletal muscle cells, this does not preclude the possibility that excess leucine also indirectly modulates peripheral insulin sensitivity in mammals. Increased blood leucine concentrations have been associated with satiety (Cota et al. 2006; Lynch et al. 2006) and increased energy expenditure (Zhang et al. 2007), the latter likely due to its potent effects at stimulating tissue protein synthesis, an energy intensive process. Indeed, doubling dietary leucine intake in mice has been associated with decreasing adiposity in diet-induced obese animals while improving whole-body insulin sensitivity (Zhang et al. 2007). Given the widely described role of adiposity in the development of insulin resistance, leucine could be exerting effects in an indirect manner. Likewise, the possibility that metabolites of leucine or amino acids other than leucine contribute to perturbed glucose uptake upon exogenous leucine administration cannot be excluded. It is widely acknowledged that the bolus administration of leucine results in increased formation of leucine metabolites (Lee et al. 2015) and a reciprocal fall in plasma concentrations of the other branched chain amino acids (Matsumoto et al. 2014). Notably, both the leucine metabolite $\alpha$-ketoisocaproate and the branched chain amino acid isoleucine have been shown to stimulate increased glucose uptake in muscle (Doi et al. 2007; Nishitani et al. 2002) and cultured myotubes (Doi et al. 2003). Crucially, utilizing cultured cells, we circumvented the issues associated with the impact of leucine on distal tissues and systems, and by applying a multipronged approach to modulate leucine transport and availability, we demonstrated that the amino acid exerts a direct effect on glucose uptake in muscle cells, albeit the lack of a direct measure of intracellular free leucine concentrations is a limitation of the current study.

While our findings arguably suggest that leucine administration could offer beneficial effects by improving insulin sensitivity via increasing insulin-dependent muscle glucose uptake, trials of chronic leucine supplementation in type-2 diabetic men have proved disappointing to date. A study examining the consumption of $7.5 \mathrm{~g} /$ day of leucine 
for 6 months taken in addition to the subject's habitual diet failed to show any metabolic or anthropometric benefit to diabetic patients, with insulin sensitivity, as determined by oral glucose tolerance test, unaffected by the intervention (Leenders et al. 2011). Although leucine supplementation may ultimately prove to be an ineffective treatment strategy, our data revealed that targeting the mTORc1 signalling pathway remains judicious. In particular, we observed that the mTORc1 activator, 3BDO, has a profound ability to increase glucose uptake in a non-insulin-dependent manner. Contrary to expectations, the beneficial effects of 3BDO did not appear due to increased mTORc1 signalling, suggesting that its effects occur via alternative mechanisms. Indeed, in L6 myotubes, 3BDO appeared to inhibit the phosphorylation of mTORc1 and its downstream targets despite the administration of a dose of 3BDO known to increase mTORc1 activity in other cell types (Ge et al. 2014). Whether this is a response specific to muscle cells, the dose utilized, or the timepoint examined is unclear, but regardless, our data suggest that the 3BDO compound is worthy of further investigation, particularly in the quest for strategies to aid glucose homeostasis in diabetic patients.

\section{Conclusions}

In summary, results from this study demonstrate that there is a relationship between mTORc1 activity and glucose uptake in L6 myotubes, but this appears independent of the phosphorylation status of AKT. Similarly, while leucine promotes basal and insulin-induced glucose uptake in L6 myotubes, this occurs independent of AKT-mTORc1 signalling. Collectively, our findings reinforce the direct role that leucine plays in the regulation of muscle carbohydrate metabolism and suggests that strategies aimed at reducing obesity-induced hyperleucinaemia to improve peripheral insulin sensitivity could be counterproductive. However, the exact mechanism by which leucine exerts its effects on glucose uptake in muscle cells remains unknown and should be the focus of future work.

Funding This study was funded by The University of Nottingham. QY was supported by a China Scholarship Council (CSC) Research Excellence Scholarship (Scholarship Ref Number: 15595).

\section{Compliance with ethical standards}

Conflict of interest The authors declare that they have no conflict of interest.

Open Access This article is licensed under a Creative Commons Attribution 4.0 International License, which permits use, sharing, adaptation, distribution and reproduction in any medium or format, as long as you give appropriate credit to the original author(s) and the source, provide a link to the Creative Commons licence, and indicate if changes were made. The images or other third party material in this article are included in the article's Creative Commons licence, unless indicated otherwise in a credit line to the material. If material is not included in the article's Creative Commons licence and your intended use is not permitted by statutory regulation or exceeds the permitted use, you will need to obtain permission directly from the copyright holder. To view a copy of this licence, visit http://creativecommons .org/licenses/by/4.0/.

\section{References}

Balage M, Dupont J, Mothe-Satney I, Tesseraud S, Mosoni L, Dardevet D (2011) Leucine supplementation in rats induced a delay in muscle IR/PI3K signaling pathway associated with overall impaired glucose tolerance. J Nutr Biochem 22:219-226. https ://doi.org/10.1016/j.jnutbio.2010.02.001

Caballero B, Finer N, Wurtman RJ (1988) Plasma amino acids and insulin levels in obesity: response to carbohydrate intake and tryptophan supplements. Metabolism 37:672-676

Cho NH, Shaw JE, Karuranga S, Huang Y, da Rocha Fernandes JD, Ohlrogge AW, Malanda B (2018) IDF Diabetes Atlas: Global estimates of diabetes prevalence for 2017 and projections for 2045. Diabetes Res Clin Pract 138:271-281. https:// doi.org/10.1016/j.diabres.2018.02.023

Cota D, Proulx K, Smith KA, Kozma SC, Thomas G, Woods SC, Seeley RJ (2006) Hypothalamic mTOR signaling regulates food intake. Science 312:927-930. https://doi.org/10.1126/scien ce. 1124147

Deblon N et al (2012) Chronic mTOR inhibition by rapamycin induces muscle insulin resistance despite weight loss in rats. Br J Pharmacol 165:2325-2340. https://doi.org/10.111 $1 / j .1476-5381.2011 .01716 . x$

Deshmukh A, Salehzadeh F, Metayer-Coustard S, Fahlman R, Nair KS, Al-Khalili L (2009) Post-transcriptional gene silencing of ribosomal protein S6 kinase 1 restores insulin action in leucinetreated skeletal muscle. Cell Mol Life Sci 66:1457-1466. https ://doi.org/10.1007/s00018-009-8818-y

Doi M, Yamaoka I, Fukunaga T, Nakayama M (2003) Isoleucine, a potent plasma glucose-lowering amino acid, stimulates glucose uptake in C2C12 myotubes Biochem. Biophys Res Commun 312:1111-1117. DOI: 10.1016/j.bbrc.2003.11.039.

Doi M, Yamaoka I, Nakayama M, Sugahara K, Yoshizawa F (2007) Hypoglycemic effect of isoleucine involves increased muscle glucose uptake and whole body glucose oxidation and decreased hepatic gluconeogenesis. Am J Physiol Endocrinol Metab 292:E1683-1693. DOI: 10.1152/ajpendo.00609.2006.

Felig P, Marliss E, Cahill GF Jr (1969) Plasma amino acid levels and insulin secretion in obesity. N Engl J Med 281:811-816. https ://doi.org/10.1056/NEJM196910092811503

Ge D et al (2014) Identification of a novel MTOR activator and discovery of a competing endogenous RNA regulating autophagy in vascular endothelial cells. Autophagy 10:957-971. https:// doi.org/10.4161/auto. 28363

Gran P, Cameron-Smith D (2011) The actions of exogenous leucine on mTOR signalling and amino acid transporters in human myotubes. BMC Physiol 11:10. https://doi. org/10.1186/1472-6793-11-10

Hurley MS, Flux C, Salter AM, Brameld JM (2006) Effects of fatty acids on skeletal muscle cell differentiation in vitro. Br J Nutr 95:623-630. https://doi.org/10.1079/bjn20051711 
Iwanaka N, Egawa T, Satoubu N, Karaike K, Ma X, Masuda S (1985) Hayashi T (2010) Leucine modulates contraction- and insulinstimulated glucose transport and upstream signaling events in rat skeletal muscle. J Appl Physiol 108:274-282. https://doi. org/10.1152/japplphysiol.00420.2009

Kleinert M, Liao YH, Nelson JL, Bernard JR, Wang W (1985) Ivy JL (2011) An amino acid mixture enhances insulin-stimulated glucose uptake in isolated rat epitrochlearis muscle. J Appl Physiol 111:163-169. https://doi.org/10.1152/japplphysiol.01368.2010

Kleinert $\mathrm{M}$ et al (2014) Acute mTOR inhibition induces insulin resistance and alters substrate utilization in vivo. Mol Metab 3:630-641. https://doi.org/10.1016/j.molmet.2014.06.004

Lee AJ et al (2015) A (14)C-leucine absorption, distribution, metabolism and excretion (ADME) study in adult Sprague-Dawley rat reveals beta-hydroxy-beta-methylbutyrate as a metabolite. Amino Acids 47:917-924. https://doi.org/10.1007/s0072 6-015-1920-6

Leenders $\mathrm{M}$ et al (2011) Prolonged leucine supplementation does not augment muscle mass or affect glycemic control in elderly type 2 diabetic men. J Nutr 141:1070-1076. https://doi.org/10.3945/ jn.111.138495

Li X et al (2013) Chronic leucine supplementation increases body weight and insulin sensitivity in rats on high-fat diet likely by promoting insulin signaling in insulin-target tissues. Mol Nutr Food Res 57:1067-1079. https://doi.org/10.1002/mnfr.201200311

Liu $\mathrm{H}$ et al (2014) Leucine facilitates the insulin-stimulated glucose uptake and insulin signaling in skeletal muscle cells: involving mTORC1 and mTORC2. Amino Acids 46:1971-1979. https:// doi.org/10.1007/s00726-014-1752-9

Lynch CJ, Gern B, Lloyd C, Hutson SM, Eicher R, Vary TC (2006) Leucine in food mediates some of the postprandial rise in plasma leptin concentrations. Am J Physiol Endocrinol Metab 291:E621630. https://doi.org/10.1152/ajpendo.00462.2005

Matsumoto T et al (2014) Bolus ingestion of individual branched-chain amino acids alters plasma amino acid profiles in young healthy men. Springerplus 3:35. https://doi.org/10.1186/2193-1801-3-35

Moghei M, Tavajohi-Fini P, Beatty B, Adegoke OA (2016) Ketoisocaproic acid, a metabolite of leucine, suppresses insulin-stimulated glucose transport in skeletal muscle cells in a BCAT2-dependent manner. Am J Physiol Cell Physiol 311:C518-527. https://doi. org/10.1152/ajpcell.00062.2016

Mubeen U, Juppner J, Alpers J, Hincha DK, Giavalisco P (2018) Target of rapamycin inhibition in Chlamydomonas reinhardtii triggers de novo amino acid synthesis by enhancing nitrogen assimilation. Plant Cell 30:2240-2254. https://doi.org/10.1105/tpc.18.00159

Murton AJ, Constantin D, Greenhaff PL (2008) The involvement of the ubiquitin proteasome system in human skeletal muscle remodelling and atrophy. Biochim Biophys Acta 1782:730-743. https:// doi.org/10.1016/j.bbadis.2008.10.011

Murton AJ, Alamdari N, Gardiner SM, Constantin-Teodosiu D, Layfield R, Bennett T, Greenhaff PL (2009) Effects of endotoxaemia on protein metabolism in rat fast-twitch skeletal muscle and myocardium. PLoS ONE 4:e6945. https://doi.org/10.1371/journ al.pone. 0006945

Murton AJ, Marimuthu K, Mallinson JE, Selby AL, Smith K, Rennie MJ, Greenhaff PL (2015) Obesity appears to be associated with altered muscle protein synthetic and breakdown responses to increased nutrient delivery in older men, but not reduced muscle mass or contractile function. Diabetes 64:3160-3171. https://doi. org/10.2337/db15-0021
Newgard CB et al (2009) A branched-chain amino acid-related metabolic signature that differentiates obese and lean humans and contributes to insulin resistance. Cell Metab 9:311-326. https://doi. org/10.1016/j.cmet.2009.02.002

Nishitani S, Matsumura T, Fujitani S, Sonaka I, Miura Y, Yagasaki K (2002) Leucine promotes glucose uptake in skeletal muscles of rats. Biochem Biophys Res Commun 299:693-696. https://doi. org/10.1016/s0006-291x(02)02717-1

Peng N et al (2014) An activator of mTOR inhibits oxLDL-induced autophagy and apoptosis in vascular endothelial cells and restricts atherosclerosis in apolipoprotein E(-)/(-) mice. Sci Rep 4:5519. https://doi.org/10.1038/srep05519

Poncet $\mathrm{N}$ et al (2014) The catalytic subunit of the system L1 amino acid transporter (slc7a5) facilitates nutrient signalling in mouse skeletal muscle. PLoS ONE 9:e89547. https://doi.org/10.1371/ journal.pone.0089547

Saha AK, Xu XJ, Lawson E, Deoliveira R, Brandon AE, Kraegen EW, Ruderman NB (2010) Downregulation of AMPK accompanies leucine- and glucose-induced increases in protein synthesis and insulin resistance in rat skeletal muscle. Diabetes 59:2426-2434. https://doi.org/10.2337/db09-1870

Sarabia V, Ramlal T, Klip A (1990) Glucose uptake in human and animal muscle cells in culture. Biochem Cell Biol 68:536-542

Sheng YL et al (2017) Urate promotes SNCA/alpha-synuclein clearance via regulating mTOR-dependent macroautophagy. Exp Neurol 297:138-147. https://doi.org/10.1016/j.expneurol.2017.08.007

Wang X et al (2016) Rheb1-mTORC1 maintains macrophage differentiation and phagocytosis in mice. Exp Cell Res 344:219-228. https://doi.org/10.1016/j.yexcr.2016.04.017

White PJ, Newgard CB (2019) Branched-chain amino acids in disease. Science 363:582-583. https://doi.org/10.1126/science.aav0558

White PJ et al (2018) The BCKDH kinase and phosphatase integrate BCAA and lipid metabolism via regulation of ATP-citrate lyase. Cell Metab 27(1281-1293):e1287. https://doi.org/10.1016/j. cmet.2018.04.015

Wolfson RL, Chantranupong L, Saxton RA, Shen K, Scaria SM, Cantor JR, Sabatini DM (2016) Sestrin2 is a leucine sensor for the mTORC1 pathway. Science 351:43-48. https://doi.org/10.1126/ science.aab2674

Xiao F et al (2011) Leucine deprivation increases hepatic insulin sensitivity via GCN2/mTOR/S6K1 and AMPK pathways. Diabetes 60:746-756. https://doi.org/10.2337/db10-1246

Yap A, Nishiumi S, Yoshida K, Ashida H (2007) Rat L6 myotubes as an in vitro model system to study GLUT4-dependent glucose uptake stimulated by inositol derivatives. Cytotechnology 55:103108. https://doi.org/10.1007/s10616-007-9107-y

Yoneyama $\mathrm{Y}$ et al. (2018) Serine phosphorylation by mTORC1 promotes IRS-1 degradation through SCFbeta-TRCP E3 ubiquitin ligase. iScience 5:1-18. DOI: 10.1016/j.isci.2018.06.006.

Zhang Y, Guo K, LeBlanc RE, Loh D, Schwartz GJ, Yu YH (2007) Increasing dietary leucine intake reduces diet-induced obesity and improves glucose and cholesterol metabolism in mice via multimechanisms. Diabetes 56:1647-1654. https://doi.org/10.2337/ db07-0123

Publisher's Note Springer Nature remains neutral with regard to jurisdictional claims in published maps and institutional affiliations. 